\title{
Calculation of the Modified Bessel Functions of the Second Kind with Complex Argument
}

\author{
By Fr. Mechel
}

In physical problems often the modified Bessel functions $K_{n}(z)$ with complex argument $z$ occur, or the Hankel functions of the first kind $H_{n}{ }^{(1)}(z)$ which are related to $K_{n}(z)$ by $K_{n}(z)=(\pi / 2) i^{n+1} H_{n}{ }^{(1)}(i z)$, with $i=(-1)^{1 / 2}$. Computationally rapid and fairly exact procedures are available for the evaluation of these functions for real or imaginary argument either by recurrence, [1], [2], or by polynomial approximations, [3]-[7]. With complex argument, methods of computation were published which start from the representation by integrals of the modified Bessel function, [8], [9], [10].

Hunter [10] makes use of the integral

$$
K_{n}(z)=\frac{\pi^{1 / 2} e^{-z}}{\Gamma\left(n+\frac{1}{2}\right)(2 z)^{n}} \int_{-\infty}^{+\infty} \exp \left[-t^{2}\right] t^{2 n}\left(2 z+t^{2}\right)^{n-1 / 2} d t
$$

valid for $|\arg z|<\pi$. The integration can be approximated by summation according to the trapezoidal rule. This representation, however, fails for small $|z|(|z|<2$, say), both because of the increase of the integration error and because $z$ in the integrand enters as a summand. Whereas, on the other hand, the evaluation according to

$$
K_{n}(z)=\int_{0}^{\infty} \exp [-z \cosh t] \cosh n t d t, \quad|\arg z|<\pi / 2
$$

proposed by Fettis [8] and Luke [9] has its maximum accuracy for small $|z|$, the restriction on the argument, however, for many applications is too severe, the more so as the computation will diverge near the imaginary axis. In a trial computation this range of divergence could not even be eliminated by a polynomial approximation near the imaginary axis according to [6].

These drawbacks are avoided with the integral representation [11], [12], discussed in this paper:

$$
\begin{aligned}
& K_{n}(z)=-\frac{i}{(2 \pi)^{3 / 2}} \cdot \cos n \pi \cdot \frac{e^{-z}}{z^{1 / 2}} \\
& \cdot \int_{-i \infty}^{+i \infty} \Gamma(s) \Gamma\left(\frac{1}{2}-s-n\right) \Gamma\left(\frac{1}{2}-s+n\right)(2 z)^{s} d s
\end{aligned}
$$

which is valid for $|\arg z|<\frac{3}{2} \pi$ and $2 n$ no odd integer. The path of integration must separate the poles at $s=-m,(m=0,1,2)$ of the first gamma function $\Gamma(s)$ from the poles at $s=\frac{1}{2}+m-n$ and $s=\frac{1}{2}+m+n$ of the other gamma function factors. For the special cases $n=0$ and $n=1$ the poles and the paths $L_{n}$ of integration are represented in Figure 1.

With $n=1$ the integrand has the residue $(2 \pi / z)^{1 / 2}$ at the pole $s=-\frac{1}{2}$. With

Received September 7, 1965. 

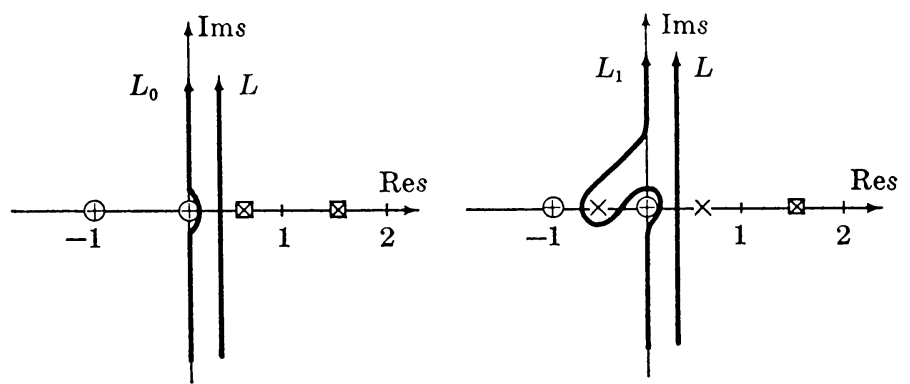

Figure 1. Paths of integration $L_{n}$ and poles of (3) for $n=0$ and $1 . \oplus$ pole of $\Gamma(s), \times$ pole of $\Gamma\left(\frac{1}{2}-s-n\right), \otimes$ pole of $\Gamma\left(\frac{1}{2}-s+n\right)$.

the theorem of residues the paths $L_{n}$ can be transformed into the one path $L$ parallel to the imaginary axis in a distance $s=\frac{1}{4}$. After transformation of the variable of integration $s=\frac{1}{4}+i t$ and using the recurrence relation of the gamma function we finally obtain for $K_{0}(z)$ and $K_{1}(z)$ the representations

$$
\begin{aligned}
& K_{0}(z)=\frac{e^{-z}}{z^{1 / 2}} \cdot \frac{(2 z)^{1 / 4}}{(2 \pi)^{3 / 2}} \int_{-\infty}^{+\infty} \Gamma\left(\frac{1}{4}+i t\right) \Gamma^{2}\left(\frac{1}{4}-i t\right)(2 z)^{i t} d t \\
& K_{1}(z)=\frac{e^{-z}}{z^{1 / 2}} \cdot\left[\frac{1}{z^{1 / 2}}-\frac{(2 z)^{1 / 4}}{(2 \pi)^{3 / 2}} \int_{-\infty}^{+\infty} \Gamma\left(\frac{1}{4}+i t\right) \Gamma^{2}\left(\frac{1}{4}-i t\right) \frac{4 i t-1}{4 i t+3}(2 z)^{i t} d t\right] .
\end{aligned}
$$

For the numerical evaluation this representation has several advantages:

(1) In the simply covered $z$-plane there is no limitation of the validity outside of the singular point $z=0$ provided $z$, in the computation, is written $z=r \cdot e^{i \phi}$ with $\phi$ restricted to $|\phi| \leqq \pi$.

(2) The argument $z$ enters as a factor into the integrand. This factor is $(2 z)^{i t}=e^{-t \phi}[\cos (t \cdot \ln 2 r)+i \sin (t \cdot \ln 2 r)]$. Large absolute values $r \gg 1$ and small ones $r \ll 1$ differ from one another only by the sense of rotation of the factor in the brackets. Another advantage is the fact that $r$ appears only with its logarithm thus avoiding the exceeding of the argument range of library subroutines of the cos and sin functions in electronic computers.

(3) The absolute value of both of the integrands converges for $|t| \rightarrow \infty$ towards $(2 \pi)^{3 / 2} \cdot|e t|^{-3 / 4} \cdot \exp \left(-\frac{3}{2} \pi|t|-t \phi\right)$. The rate of convergence of the integrands is different on the two branches of the path $L$ on both sides of $t=0$ by the factor $\exp ( \pm t \phi)$. In the simply covered $z$-plane $|\phi| \leqq \pi$. So even on the "slow" branch the convergence is as good as $|t|^{-3 / 4} \cdot \exp (-(\pi / 2)|t|)$.

(4) The factor $\Gamma\left(\frac{1}{4}+i t\right) \Gamma^{2}\left(\frac{1}{4}-i t\right)$ can be stored as system constants at the points of integration. This factor is conjugate complex on the two branches of the integration path. Further, $\Gamma\left(\frac{1}{4}+i t\right)$ and $\Gamma\left(\frac{1}{4}-i t\right)$ are the conjugates of one another. So the gamma function needs only be evaluated for one branch.

The gamma function can be easily computed from its asymptotic expansion

$$
\begin{aligned}
\ln \Gamma(z) & =\frac{1}{2} \ln 2 \pi+\left(z-\frac{1}{2}\right) \ln z-z+\psi(z), \quad \text { for }|z| \gg 1 \\
\psi(z) & =\sum_{k=1}^{n-1} \frac{B_{2 k}}{2 k(2 k-1) z^{2 k-1}}+R_{n}(z), \\
\left|R_{n}(z)\right| & <\frac{\left|B_{2 n}\right|}{2 n(2-1)|\operatorname{Re} z|^{2 n-1}}
\end{aligned}
$$


$B_{2 k}$ the Bernoulli numbers. In [13] $\psi(z)$ is written in the form $\psi(z)=\sum_{k=1}^{10} A_{k} / z^{2 k-1}$ and the $A_{k}$ are tabulated to 25 significant figures. For $\operatorname{Re} z>10$ the error on the abscissa (where it is greatest) is $R_{11}(z)<1.4 \cdot 10^{-19}$. The function $\Gamma(z)$ for $0<\operatorname{Re} z \leqq 10$ can be evaluated with the recursion formula of the gamma function.

(5) Finally, the integrands are well suited for integration with the trapezoidal rule. This will become evident by the succeeding estimation of the error of integration. Two components of the error should be distinguished: the error due to the truncation of the summation in the trapezoidal rule and the error inherent to the trapezoidal rule itself.

The truncation errors $E_{T}\left(M_{ \pm}\right)$made by a truncation of the summation after $M_{+}-1$ in the "fast" branch of integration or after $M_{-}-1$ in the "slow" branch are discussed in the appendix. They are of the order of magnitude of

$$
\left|E_{T}\left(M_{ \pm}\right)\right| \lesssim\left|T_{M_{ \pm}}\right| e^{1 / 4}\left(1+1 / 4 M_{ \pm} h\right)(h / 2+1 /(3 \pi / 2 \pm|\phi|))
$$

where $T_{M_{ \pm}}$is the first neglected term in the sums and $h$ is the step width of the trapezoidal rule. The truncation error is of the order of magnitude of the first neglected term in the sum.

To get a bound for the error of the integration of (4) and (5) inherent to the trapezoidal rule we make use of Poisson's summation formula, [14],

$$
\int_{-\infty}^{+\infty} f(x) d x=h \sum_{k=-\infty}^{+\infty} f(k h)+\sum_{k \neq 0} \int_{-\infty}^{+\infty} f(x) \exp [i 2 \pi k x / h] d x .
$$

Here the first term is just the trapezoidal rule and the second term is the error $E$. For application of (6) we write in (4) and (5) the integrals

$$
F_{n}(z)=\int_{-\infty}^{+\infty} G_{n}(t, z) d t=\int_{-\infty}^{+\infty} g_{n}(t, \phi) \exp [i t \cdot \ln 2 r] d t, \quad n=0,1 .
$$

Then the error is

$$
\begin{aligned}
E_{n}(h, z) & =\sum_{k \neq 0} \int_{-\infty}^{+\infty} g_{n}(t, \phi) \exp [i t(\ln 2 r+2 \pi k / h)] d t \\
& =\sum_{k \neq 0} \int_{-\infty}^{+\infty} g_{n}(t, \phi) \exp \left[i t \cdot \ln \left(2 r e^{2 \pi k / h}\right)\right] d t \\
& =\sum_{k \neq 0} F_{n}\left(e^{2 \pi k / h} \cdot z\right) .
\end{aligned}
$$

$F_{n}(z)$ can be obtained from (4) or (5):

$$
F_{n}(z)=2^{5 / 4} \pi^{3 / 2} e^{z} z^{1 / 4}\left[\delta_{n, 1} \cdot z^{-1 / 2}+(-1)^{n} K_{n}(z)\right] .
$$

With small steps of integration $h$ the terms in the sum of (8) will contain, for $k>0$, modified Bessel functions with large absolute values of the argument and, for $k<0$, those with small absolute values of the argument. Therefore, the approximations

$$
K_{n}(z) \approx\left(\frac{\pi}{2}\right)^{1 / 2} e^{-z} z^{-1 / 2} \quad \text { for }|z| \gg 1,
$$

and

$$
K_{0}(z) \approx \ln \frac{2}{\gamma}-\ln z ; \quad K_{1} \approx \frac{1}{z} \quad \text { for }|z| \ll 1
$$

( $\gamma=1.781 \cdots$, Euler's constant) can be used. 
Then after some computation and dropping small terms we have for the error of integration for $K_{0}(z)$ :

$$
E_{0}(h, z) \approx \frac{2 \pi^{2}}{(2 z)^{1 / 4}} \sum_{k=1}^{\infty} e^{-k \pi / 2 h}=\frac{2 \pi^{2}}{(2 z)^{1 / 4}} \frac{e^{-\pi / 2 h}}{1-e^{-\pi / 2 h}}
$$

and for the integration of $K_{1}(z)$ :

$$
E_{1}(h, z) \approx(2 \pi)^{3 / 2}\left[(z / 2)^{1 / 4}-\left(\frac{\pi}{2}\right)^{1 / 2}(2 z)^{-1 / 4}\right] \frac{e^{-\pi / 2 h}}{1-e^{-\pi / 2 h}} .
$$

These formulas again reflect the small influence of the order of magnitude of $|z|$ on the accuracy of the integration.

The formulas (4) and (5) were used for the computation of $K_{0}(z)$ and $K_{1}(z)$ on an IBM 7040 computer with double precision (17D). The trapezoidal rule was applied. The results were checked on the axes of the $z$-plane against a program (after [3]) with 15 decimals accuracy. With a step $h$ of 0.05 the accuracy on the positive real axis and on the imaginary axis was 10 to 15 decimals. The smaller value belongs to large imaginary arguments. The accuracy on the negative real axis was better than 5 decimals. Here the summation did not go far enough to obtain the full accuracy predicted by $(9)$ and $(10)$.

As a trial the integration of (4) and (5) was performed also with use of Simpson's formula. With equal step $h$ this resulted in a loss of about 4 decimals of accuracy.

The computation of the modified Bessel functions using (4) and (5) needs smaller steps $h$ than with the formulas (1) and (2). This is the price for the greater range of validity of the argument $z$. However, the computational work can be reduced if we exploit the facts mentioned in item (4).

For explanation we denote with $u_{n}(t)$ the $z$-independent factors in the integrands of (4) and (5) (where at least the factor with the gamma functions is stored as a system constant). We further write the integrands

$$
G_{n}(t, z)=u_{n}(t)(2 z)^{i t}=u_{n}(t) \exp [i t \cdot \ln 2 r] e^{-t \phi}=v_{n}(t, r) e^{-t \phi}
$$

with $v_{n}(t, r)=v_{n}{ }^{\prime}+i v_{n}{ }^{\prime \prime}$ and $v_{n}(-t, r)=v_{n}{ }^{*}(t, r)$ (where ${ }^{*}$ denotes conjugate complex). Then the integrals are

$$
F_{n}(z)=\int_{0}^{\infty}\left[v_{n}{ }^{\prime} e^{|\phi| t}\left(1+e^{-2|\phi| t}\right) \mp i \cdot v_{n}{ }^{\prime \prime} e^{|\phi| t}\left(1-e^{-2|\phi| t}\right)\right] d t
$$

where the upper sign in the brackets holds for $\phi>0$ and the lower sign for $\phi<0$ whereas for $\phi=0$ only $2 v_{n}{ }^{\prime}$ remains under the integral. With the trapezoidal rule (11) becomes

$$
\begin{aligned}
F_{n}(z)=h \cdot\left[\epsilon_{n} \Gamma^{3}\left(\frac{1}{4}\right)+\sum_{k=1}^{M} v_{n}{ }^{\prime}(k h, r) e^{|\phi| k h}\left(1+e^{-2|\phi| k h}\right)\right. & \\
& \left.\mp i \cdot v_{n}{ }^{\prime \prime}(k h, r) e^{|\phi| k h}\left(1-e^{-2|\phi| k h}\right)\right]
\end{aligned}
$$

with $\epsilon_{0}=1$ and $\epsilon_{1}=-\frac{1}{3}$. The upper summation limit $M$ is determined by the number $N$ of exact decimals that are desired. $M$ is given by

$$
M=N /[h(\log e)(1.5 \pi-|\phi|)] .
$$


Further, the terms $\left(1 \pm e^{-2|\phi| k h}\right)$ can be replaced by unity for $k \geqq M_{1}$ with $M_{1}=$ $N_{1} /[2 h(\log e)(1.5 \pi+|\phi|)]$ where $N_{1}$ is the number of significant digits in the computation. Finally, the factor $\exp [j k h \cdot \ln r]$ can be computed by iterated application of the addition theorem of the trigonometric functions. In order to control the increase of the multiplication error in this term it is advisable, however, to compute it anew after an interval $\Delta k$ (of $\Delta k=10$, say). A similar procedure for $e^{|\phi| k h}$ would destroy the accuracy. With these simplifications the operations that remain are mainly the evaluation of $e^{|\phi| k h}$ and complex multiplications.

\section{ApPENDIX}

Estimation of the Truncation Error. In the trapezoidal rule (6) the summation index $k$ is assumed to run to $\pm \infty$. In practice, the restriction to a finite limit for $k$ brings a truncation error $E_{T}$ into the result. In contrast to (12) where the same upper limit $M$ of the summation index $k$ was used for both the "fast" and the "slow" branch of integration we now assume the absolute value of $k$ to go to $M_{+}-1$ and $M_{-}-1$ in these branches respectively. The truncation error of the sum then is $E_{T}=E_{T}\left(M_{+}\right)+E_{T}\left(M_{-}\right)$. For simplicity, we restrict ourselves to the error of $K_{0}$. However, for $|k h| \geqq M_{ \pm} h \gg 1$ the absolute value of the fraction in the integrand of $K_{1}$ approaches unity, so the result holds for $K_{1}$ too.

For $|t| \gg 1$ the absolute value of the integrand $G_{0}(t, z)$ can be approximated by

$$
\left|G_{0}(t, z)\right| \approx(2 \pi)^{3 / 2} e^{-1 / 2} \exp [-3 \pi|t| / 2]|t|^{-3 / 4}(1+(1 / 4|t|)) e^{-t \phi}
$$

where the asymptotic expansion of the gamma function and the approximation

$$
\left|\arg \left(\frac{1}{4}+i t\right)\right| \approx \frac{\pi}{2}-\frac{1}{4 t}+\frac{1}{192 t^{3}}-\cdots
$$

were used with the first two terms.

With this and with the notations $\alpha=(2 \pi)^{3 / 2} e^{-1 / 2}$ and $T_{ \pm}=M_{ \pm} h$ the truncation error becomes

$$
\begin{aligned}
&\left|E_{T}\left(M_{ \pm}\right)\right| \lesssim \alpha \exp \left[-T_{ \pm}\left(\frac{3 \pi}{2} \pm \phi\right)\right] h \sum_{k=0}^{\infty}\left(k h+T_{ \pm}\right)^{-3 / 4} \\
& \cdot\left(1+\frac{1}{4\left(k h+T_{ \pm}\right)}\right) \exp \left[-k h\left(\frac{3 \pi}{2}+\phi\right)\right] .
\end{aligned}
$$

The sum can be interpreted as an approximation to an integral. (The error of this approximation is of the same order of magnitude as the error of the trapezoidal rule when applied to $K_{0}$.) The integral leads to the Whittaker function $W_{\lambda, \mu}(x)$. Therefore, we have

$$
\begin{aligned}
\left|E_{T}\left(M_{ \pm}\right)\right| \lesssim \alpha & \exp \left[-T_{ \pm}(3 \pi / 2 \pm \phi)\right]\left[\frac{h}{2}\left(T_{ \pm}^{-3 / 4}+\frac{1}{4} T_{ \pm}^{-7 / 4}\right)\right. \\
+T_{ \pm}^{-3 / 8}\left(\frac{3 \pi}{2} \pm \phi\right)^{-5 / 8} W_{-3 / 8,1 / 8}\left(T_{ \pm}\left(\frac{3 \pi}{2} \pm \phi\right)\right) & \\
& \left.+\frac{1}{4} T_{ \pm}^{7 / 8}\left(\frac{3 \pi}{2} \pm \phi\right)^{-1 / 8} W_{-7 / 8,-3 / 8}\left(T_{ \pm}\left(\frac{3 \pi}{2} \pm \phi\right)\right)\right] .
\end{aligned}
$$


For our application, the argument of the Whittaker function is large enough to use the asymptotic expansion of the function. Then we have:

$$
\begin{aligned}
& \left|E_{T}\left(M_{ \pm}\right)\right| \lesssim(2 \pi)^{3 / 2} e^{-1 / 2} \exp \left[-T_{ \pm}\left(\frac{3 \pi}{2} \pm \phi\right)\right] T_{ \pm}{ }^{1 / 4}\left[\frac{h}{2}\left(\frac{1}{T_{ \pm}}+\frac{1}{4 T_{ \pm}^{2}}\right)\right. \\
& \left.\quad+\left(1+\frac{1}{4 T_{ \pm}}\right)\left[T_{ \pm}\left(\frac{3 \pi}{2} \pm \phi\right)\right]^{-1}-\left(\frac{3}{4}+\frac{7}{16 T_{ \pm}}\right)\left[T_{ \pm}\left(\frac{3 \pi}{2} \pm \phi\right)\right]^{-2}+\cdots\right] .
\end{aligned}
$$

If we separate the first neglected term of the sum and omit the quadratic term in the formula above we obtain the estimate of the truncation error quoted in item (5) of the text.

III. Physikalisches Institut

Universität Göttingen

Germany

1. I. A. Stegrn \& M. Abramowitz, "Generation of Bessel functions on high speed computers," MTAC, v. 11, 1957, pp. 255-257. MR $20 \# 459$.

2. M. Goldstein \& R. M. Thaler, "Recurrence techniques for the calculation of Bessel functions," MTAC, v. 13, 1959, pp. 102-108. MR 21 \#4530.

3. C. W. Clenshaw, Chebyshev Series for Mathematical Functions, Nat. Phys. Lab. Math. Tables, Vol. 5, Dept. Scientific and Indust. Research, Her Majesty's Stationery Office, London, 1962. MR $26 \# 362$.

4. C. HAStings, JR. \& J. P. Wong, JR., "Analytical approximations," MTAC, v. 8, 1954,

5. A. J. M. HIтсHCOCK, "Polynomial approximations to Bessel functions of order zero and one and to related functions," MTAC, v. 11, 1957, pp. 86-188. MR 19, 64 .

6. J. Wimp, "Polynomial expansions of Bessel functions and some associated functions," Math. Comp., v. 16, 1962, pp. 446-458. MR 26 \#6452.

7. F. D. Burgoyne, "Polynomial approximations to $I_{0}(x), I_{1}(x)$ and related functions," Math. Comp., v. 16, 1962, pp. 497-498. MR 26 \#5196.

8. H. E. FeTTIS, "Numerical calculation of certain definite integrals by Poisson's summation formula," MTAC, v. 9, 1955, pp. 85-92. MR 17, 302.

9. Y. L. LCKE, "Simple formulas for the evaluation of some higher transcendental functions," J. Wath. Phys., v. 34, 1956, pp. 298-307. MR 17, 1138.

10. D. B. Hunter, "The calculation of certain Bessel functions," Math. Comp., v. 18, 1964, pp. 123-128. MR 28 \#1330.

11. Wilhelm Magnus \& Fritz Oberhettinger, Formeln und Sätze für die speziellen Funktionen der mathematischen Physik, Springer, Berlin, 1943; 2nd ed., 1948; English transl., Chelsea, New York, 1949. MR 9, 183, MR 10, 38, 532.

12. I. M. RYŽIK \& I. S. GRADŠTEIN, Tables of integrals, sums, series and products, 3rd ed., GITTL, Moscow, 1951; 4th ed., Fizmatigiz, Moscow, 1963; English and German transl. of 3rd ed., Summen-, Produkt- und Integral-tafeln, VEB Deutscher Verlag der Wissenschaften, Berlin, 1957 ; 2nd rev. ed. of transl., VEB Deutscher Verlag der Wissenschaften, Berlin and Plenum Press, New York, 1963, Formulas 7.323 and 7.335. MR 14, 643; MR $22 \# 3120$; MR $28 \# 1326$.

13. H. Werner \& R. Collinge, "Chebyshev approximations to the gamma function," Math. Comp., v. 15, 1961, p. 195 .

14. E. Madelung, Die mathematischen Hilfsmittel des Physikers, 4th ed., Die Grundlehren der mathematischen Wissenschaften, Bd. 4, Springer, Berlin, 1950; 7th ed., 1964, p. 69. MR 12, 484. MR $29 \# 1773$. 